\title{
Deserción académica en educación general básica superior en la ciudad de Piñas y la intervención del trabajador social
}

\author{
Arelys Monserrat Escandón Barnuevo \\ arelys.escandon@unl.edu.ec \\ Universidad Nacional de Loja \\ Betti del Cisne Reyes Masa \\ betti.reyes@unl.edu.ec \\ Universidad Nacional de Loja \\ Celsa Beatriz Carrión Berrú \\ celsa.carrion@unl.edu.ec \\ Universidad Nacional de Loja \\ Johvana Aguirre Mendoza \\ johvana.aguirre@unl.edu.ec \\ Universidad Nacional de Loja
}

\section{RESUMEN}

La siguiente investigación tiene como objetivo describir los factores que influyen en la deserción educativa de los estudiantes de Educación General Básica Superior de la Ciudad de Piñas durante el periodo 2020 -2021. Esta investigación se realizó en tres fases: Investigación Bibliográfica; Determinación de métodos, instrumentos y muestra de la población; Aplicación y análisis de estrategias de recolección de datos La muestra intencionada bordean un $5 \%$ de la población de la educación general básica en un determinado colegio de la ciudad, identificando a 25 participantes, aparte de los docentes y autoridades. En los resultados de las encuestas la mayoría de participantes fueron mujeres; de acuerdo con ello el $43.8 \%$ de los estudiantes encuestados, viven en una familia disfuncional. Por otra parte, la vinculación académica y social de los estudiantes y representantes dentro del sistema educativo se categoriza como buena. Se identificó que el motivo de deserción académica, es que los estudiantes encuentran las tareas y clases muy agotadoras, y/o empezaron a trabajar para ayudar en el hogar. Los docentes consideran que el principal factor para que surja una deserción académica está relacionado más al factor económico que a la desintegración familiar, además que, la estabilidad psicológica del estudiante se ve afectada por las expectativas y demandas de los padres o representantes y por influencia de terceros.

Palabras Clave: deserción académica; factores socioeconómicos; factores familiares. 


\title{
Academic desertion in higher general basic education in the city of Piñas and the intervention of the social worker
}

\begin{abstract}
The following research aims to describe the factors that influence the educational desertion of students of General Basic Higher Education in the city of Piñas during the period 2020 -2021. This research was conducted in three phases: Bibliographic research; Determination of methods, instruments and sample of the population; Application and analysis of data collection strategies. The intentional sample is around $5 \%$ of the population of general basic education in a certain school in the city, identifying 25 participants, apart from teachers and authorities. In the results of the surveys, the majority of participants were women; accordingly, $43.8 \%$ of the students surveyed live in a dysfunctional family. On the other hand, the academic and social bonding of students and representatives within the educational system is categorized as good. It was identified that the reason for academic desertion is that students find homework and classes too exhausting, and/or started working to help at home. The teachers consider that the main factor for academic desertion is related more to the economic factor than to family disintegration, and that the psychological stability of the student is affected by the expectations and demands of the parents or representatives and by the influence of third parties.
\end{abstract}

Key Word: academic dropout; socioeconomic factors; family factors.

Artículo recibido: 05 octubre. 2021 Aceptado para publicación: 02 noviembre 2021 Correspondencia: arelys.escandon@unl.edu.ec Conflictos de Interés: Ninguna que declarar 


\section{INTRODUCCIÓN}

El presente trabajo de investigación tiene como propósito hacer un acercamiento y describir cada uno de los factores que intervienen en la toma de decisiones académicas, si bien es cierto no hay aportes directos a la educación primaria o educación general básica la mayoría de ellos son a nivel universitario, a excepción del enfoque sociológico que marca un punto importante donde surge la deserción académica. Según el Sistema de Indicadores Sociales del Ecuador, la deserción es un problema que afecta a la eficiencia del sistema educativo. El abandono escolar tiene importantes consecuencias económicas, puesto que aquellos individuos que no concluyen sus estudios se encuentran en una situación de empleo desfavorable respecto a quienes lo culminan.

(Pacho \& Chiqui, 2011)

\section{Importancia del Problema}

La deserción académica es una de las mayores problemáticas a nivel mundial; en América Latina el promedio de edad de aquellos estudiantes desertores es de 15 a 19 años, tan solo un porcentaje de estos ha completado el nivel primario de educación, este fenómeno se da más en el sector rural que en el urbano, dando como resultado que entre el $25 \%$ y el $35 \%$ de los adolescentes se veían afectados por la deserción académica. (Goicovic Donoso, 2002)

En Ecuador, en un estudio realizado durante el año 2010 al 2014, se ha visualizado un incremento en el acceso a la educación tanto en la costa como en la sierra, siendo así que el 57\% correspondía al sector costa, pero también el incremento de la deserción en la zona, siendo así el 2011 el año con mayor registro de deserción tanto en la sierra como en la costa. (Sánchez, 2015)

En el año lectivo 2019 -2020 se matricularon 2 '474.529 estudiantes de inicial a tercero de bachillerato en establecimientos fiscales, fiscomisionales, municipales y particulares del Régimen Costa. Mientras que en el 2020 - 2021 se registraron 2 '449.769 escolares de todos los sostenimientos. Por otra parte, en el Régimen Sierra las cifras aumentaron ya que en el periodo 2019-2021 se registraron 1 '862.855 matriculados mientras que en el 2020 - 2021 se registraron 1' 882.821, es decir existe un aumento de 19.966 estudiantes. (Torres, 2020) 
Al ingresar a la institución educativa, se pudo evidenciar a través de entrevistas, que la cantidad de estudiantes que desertan durante periodos "normales" es superior al que han visualizado durante el año 2020 por la pandemia, pero a pesar de ello no desaparecerá la preocupación ante la realidad que se enfrentan a diario. Actualmente en la Ciudad de Piñas, se desconoce el porcentaje total de deserción, en el Plan de Desarrollo y Ordenamiento Territorial Cantonal (2015), el 40\% de la población ha asistido o asiste al nivel de instrucción primaria y el $21.7 \%$ al nivel secundario; mientras que la deserción representa un 3.8\% en el área urbana, en el área rural está incrementó a 5.3\%; siendo así la deserción cantonal representa el $4.6 \%$ a nivel provincial, se estima que han desertado por problemas económicos, bajo rendimiento, cambio de residencia y embarazo adolescente, rara vez se ha detectado que la deserción se da por muerte prematura.

\section{METODOLOGÍA}

La investigación se enmarcará bajo el enfoque cualitativo desde el paradigma empírico analítico sociocrítico, acción que a través del investigación acción participativa, permitirá recopilar y analizar datos obtenidos de fuentes del paradigma Hermenéutico Interpretativo como la entrevista, cuestionario, grupos focales o de discusión, entre otras; con el objetivo de analizar entender y determinar los factores que influyen en la deserción de estudiantes de la ciudad de Piñas, y posteriormente realizar el respectivo plan de intervención.

El trabajo de investigación se dividió en tres fases, la primera de ellas se optó por la revisión bibliográfica que permitió la identificación y jerarquización de los factores que provocan esta toma de decisiones en el ámbito educativo; posteriormente se determinó la muestra. A cerca de la problemática y el análisis del clima estudiantil se estudió mediante encuestas de satisfacción a la población estudiantil.

La muestra de la población es no probabilística, es decir es una muestra intencionada dado que el único criterio para su elección fue estudiantes desertores y con probabilidades de deserción que bordean un 5\% de la población de la educación general básica en un determinado colegio de la ciudad, identificando a 25 participantes, aparte de los docentes y autoridades. Para confirmar la muestra se usó la siguiente regla matemática.

$$
\text { Tamaño de muestra }=\frac{\frac{z^{2} \times p(1-p)}{e^{2}}}{1+\left(\frac{z^{2} \times p(1-p)}{\left.e^{2} N\right)}\right.}
$$




\section{RESULTADO}

De acuerdo con Braxton, existen cinco categorías en los cuales se agrupan los factores de deserción, actualmente no existe una teoría respectivamente para educación general básica, las teorías estudiadas son a nivel universitario, pero se las puede implementar en todos los niveles de educación. (Himmel, 2018.)

\section{Ilustración 1. Jerarquización de Factores}

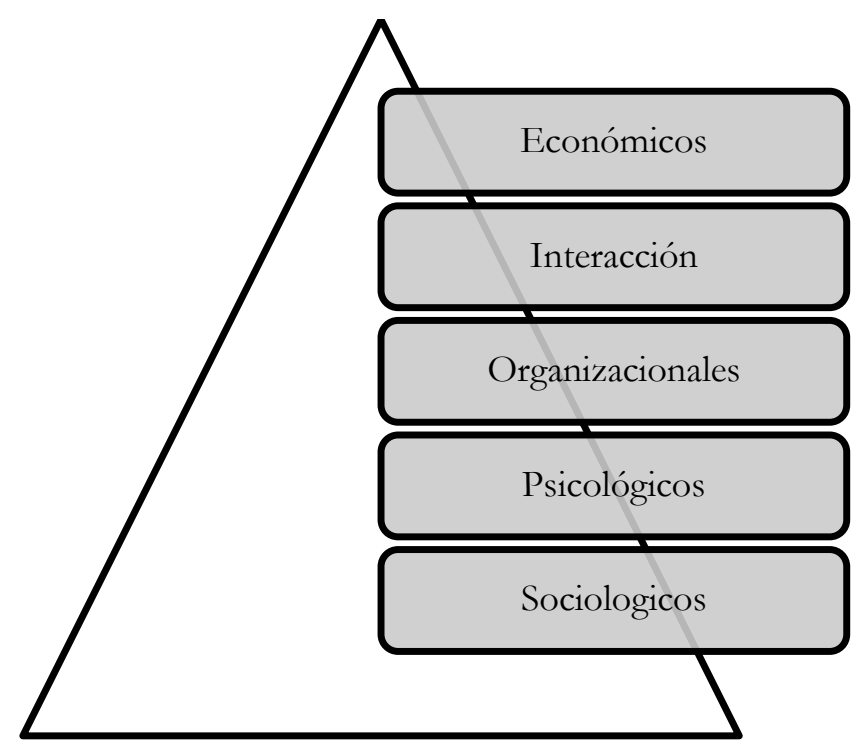

Fuente: (Himmel, 2018.), resultados de encuestas

Elaborado por: Arelys Escandón

Según Vásquez et, a partir de esto se puede diferenciar dos tipos de abandonos en estudiantes, la primera de ellas se puede denominar temporal, ya que consiste en: (Vásquez, 2003)

- Deserción precoz: cuando el estudiante ha sido aceptado en una institución educativa, pero este no se matricula.

- Deserción Temprana: Estudiante abandona sus estudios en los cuatro primeros semestres de la carrera.

- Deserción tardía: el individuo abandona los estudios en los últimos seis semestres, es decir a partir del quinto semestre.

En relación a estos el segundo tipo de deserción, se identifica respecto al espacio.

- Deserción institucional: caso en el que el estudiante abandona la institución.

- Deserción interna o del programa académico: cuando el estudiante decide cambiar por otro programa académico que ofrece la misma institución.

- Deserción del sistema Educativo. 
Dentro de los modelos sociológicos se resalta el enfoque de Jean-Claude Forquin, la relación entorno social y rendimiento académico, dado que la continuidad de preparación académica se ve afectada a partir del pase de educación general básica a básica superior, existiendo la posibilidad de la desvinculación del individuo del sistema educativo. (Cardoso \& Verner, 2011) Desde otras ramas de las Ciencias Sociales, tanto Brea como Núñez y colaboradores, manifiestan que los factores más relacionados a la deserción son factores económicos y psicoafectivos; actualmente la situación económica y migratoria del país ha afectado al núcleo familiar, fracturando la seguridad familiar y la estabilidad psicológica de cada uno de los miembros de dicha familia. (Brea de Cabral, 2005) (Sevilla-Núñez, Puerta-Chavarría, \& Dávila-Molina, 2010)

La intervención del trabajador social dentro del sistema educativo tiene como finalidad mejorar el entorno en que se desarrollan los estudiantes, ubicando a la institución como pilar de prevención y promoción de bienestar integral, es decir interviene situaciones conflictivas de los estudiantes como la drogodependencia, acoso escolar, población vulnerable ya sea por estado de salud o estado migratorio, logrando involucrarse de forma participativa a través del estudio de: situación socioeconómica, educativa. (Ministerio de Educación, 2016) (Castro Clemente \& Pérez Viejo, 2017)

Tabla 1. Género

\begin{tabular}{l|cccc} 
& \multicolumn{3}{c}{ General } & \multicolumn{2}{c}{ Desertores } \\
& $\boldsymbol{f}$ & $\boldsymbol{\%}$ & $\boldsymbol{f}$ & $\boldsymbol{\%}$ \\
\hline FEMENINO & 33 & $71.74 \%$ & 5 & $71.43 \%$ \\
\hline MASCULINO & 13 & $28.26 \%$ & 2 & $28.57 \%$ \\
\hline
\end{tabular}

Fuentes: Datos de la encuesta.

Elaborado por: Arelys Escandón

Para comprobar esto, se aplicó una encuesta tanto para estudiantes y padres de familia como para docentes, es decir participaron 46 personas en la investigación de área; siendo así solo el $34.78 \%$ fueron estudiantes menores de edad, y el $13.04 \%$ fueron docentes, dentro de esta encuesta se estudió la funcionalidad familiar, satisfacción e interacción académica y social. 
Tabla 2. Funcionalidad Familiar

\begin{tabular}{l|cccccc}
\multirow{2}{*}{} & \multicolumn{2}{c}{ Participantes } & \multicolumn{2}{c}{ Disfunción familiar } & \multicolumn{2}{c}{ Familia normal } \\
\cline { 2 - 7 } & $f$ & $\%$ & $f$ & $\%$ & $f$ & $\%$ \\
\hline Menores de edad & 16 & $40 \%$ & 7 & $17.5 \%$ & 9 & $22.5 \%$ \\
\hline Mayores de edad & 24 & $60 \%$ & 12 & $30 \%$ & 12 & $30 \%$ \\
\hline
\end{tabular}

Fuentes: Datos de la encuesta.

Elaborado por: Arelys Escandón

Estas encuestas fueron contestadas por su mayoría mujeres (71.74\%) (Tabla 1), De acuerdo con lo que expresa la Tabla 2 el $43.8 \%$ de los estudiantes encuestados, viven en una familia disfuncional. Por otra parte, la vinculación académica y social de los estudiantes y padres de familia dentro del sistema educativo se categoriza como buena. (Tabla 3, Tabla 4)

Tabla 3. Relación de estuantes con los compañeros y profesores durante su estancia en la Institución

\begin{tabular}{l|cccccccc}
\multicolumn{1}{c}{} & \multicolumn{2}{c}{ Muy buena } & \multicolumn{2}{c}{ Buena } & \multicolumn{2}{c}{ Regular } & \multicolumn{2}{c}{ Mala } \\
& $f$ & $\%$ & $f$ & $\%$ & $f$ & $\%$ & $f$ & $\%$ \\
\cline { 2 - 8 } Docentes & 16 & $40 \%$ & 23 & $57.5 \%$ & 1 & $2.5 \%$ & 0 & $0 \%$ \\
\hline Compañeros & 16 & $40 \%$ & 19 & $47.5 \%$ & 4 & $10 \%$ & 1 & $2.5 \%$ \\
\hline
\end{tabular}

Fuentes: Datos de la encuesta.

Elaborado por: Arelys Escandón

Tabla 4. Nivel de adaptación de estudiantes a la vida de la institución

\begin{tabular}{l|lccccccc}
\multicolumn{1}{c}{} & \multicolumn{3}{c}{ Muy buena } & & Buena & \multicolumn{2}{c}{ Regular } & \multicolumn{2}{c}{ Mala } \\
& $f$ & $\%$ & $f$ & $\%$ & $f$ & $\%$ & $f$ & $\%$ \\
\cline { 2 - 9 } Social & 14 & $35 \%$ & 19 & $47.5 \%$ & 6 & $15 \%$ & 1 & $2.5 \%$ \\
\hline Académica & 14 & $35 \%$ & 22 & $55 \%$ & 4 & $10 \%$ & 0 & $0 \%$ \\
\hline
\end{tabular}

Fuentes: Datos de la encuesta.

Elaborado por: Arelys Escandón

Una categoría de estudio era determinar cuál era el motivo de deserción académica, este se evaluó en dentro de cinco (5) categorías, en esta fase de la investigación participaron 7 estudiantes desertores. De esto, resaltamos los siguientes resultados por categoría: $85.71 \%$ encontró que las tareas y las clases son muy agotadores para ellos; $42.86 \%$ 
empezó a trabajar para ayudar en el hogar; 42,86\% sintió algunas veces presión por parte de los docentes; el 14,29\% sintió algunas veces presión por parte de sus compañeros; 14,29\% sintió presión de parte de su familia por el desempeño académico. (Tabla 5)

Tabla 5. Motivos de deserción académica

\begin{tabular}{|c|c|c|c|c|c|c|c|c|c|c|}
\hline & \multicolumn{2}{|c|}{ Siempre } & \multicolumn{2}{|c|}{$\begin{array}{c}\text { Casi } \\
\text { siempre }\end{array}$} & \multicolumn{2}{|c|}{$\begin{array}{l}\text { Algunas } \\
\text { veces }\end{array}$} & \multicolumn{2}{|c|}{$\begin{array}{c}\text { Casi } \\
\text { nunca }\end{array}$} & \multicolumn{2}{|c|}{ Nunca } \\
\hline & $f$ & $\%$ & $f$ & $\%$ & $f$ & $\%$ & $f$ & $\%$ & $f$ & $\%$ \\
\hline $\begin{array}{lcc}\text { Sentía presión } & \text { por } \\
\text { parte de } & \text { mis } \\
\text { compañeros } & \end{array}$ & 0 & $0 \%$ & 0 & $0 \%$ & 1 & $14.3 \%$ & 4 & $57.1 \%$ & 2 & $28.6 \%$ \\
\hline $\begin{array}{l}\text { Sentía presión por } \\
\text { parte de los docentes }\end{array}$ & 0 & $0 \%$ & 0 & $0 \%$ & 3 & $42.9 \%$ & 1 & $14.3 \%$ & 3 & $42.9 \%$ \\
\hline $\begin{array}{l}\text { Empecé a trabajar } \\
\text { para mantenerme o } \\
\text { ayudar en el hogar }\end{array}$ & 1 & $14.3 \%$ & 0 & $0 \%$ & 2 & $28.4 \%$ & 0 & $0 \%$ & 4 & $57.1 \%$ \\
\hline $\begin{array}{l}\text { Encontré que las } \\
\text { tareas y las clases } \\
\text { son muy agotadores. }\end{array}$ & 0 & $0 \%$ & 1 & $14.3 \%$ & 5 & $71.4 \%$ & 1 & $14.3 \%$ & 0 & $0 \%$ \\
\hline \begin{tabular}{l}
\multicolumn{3}{c}{ Sentía mayor presión } \\
de parte de mi \\
familia por el \\
desempeño \\
académico
\end{tabular} & 0 & $0 \%$ & 1 & $14.3 \%$ & 0 & $0 \%$ & 4 & $57.1 \%$ & 2 & $28.6 \%$ \\
\hline
\end{tabular}

Fuentes: Datos de la encuesta.

Elaborado por: Arelys Escandón

Tabla 6. Obstáculos para evitar la deserción

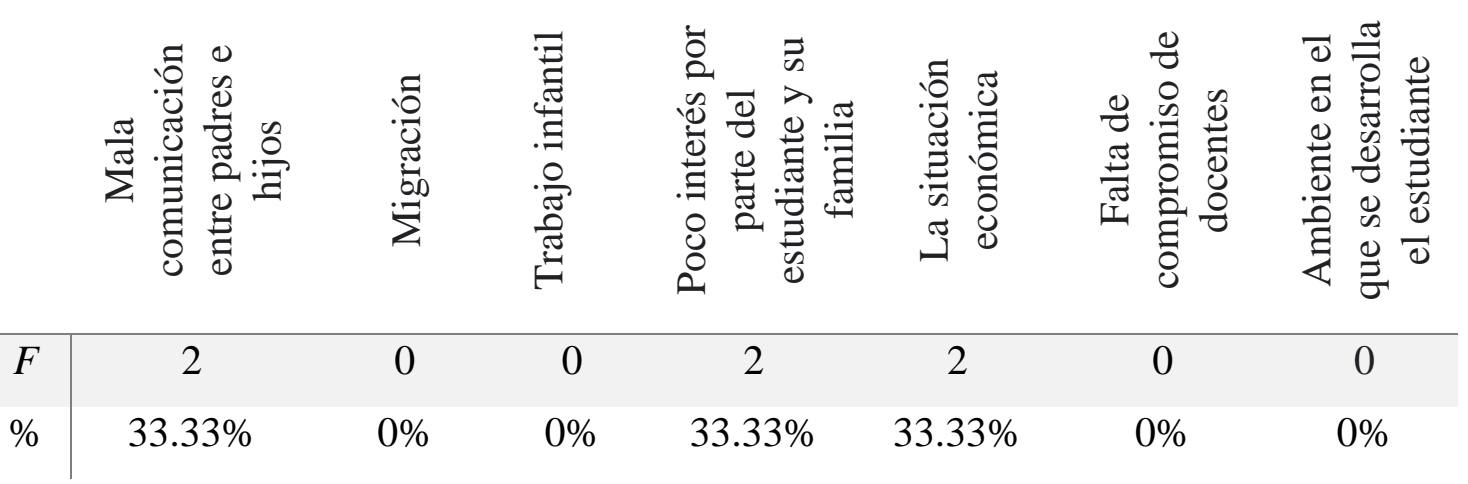

Fuentes: Datos de la encuesta.

Elaborado por: Arelys Escandón 
Por otra parte, los docentes consideran que el principal factor para que surja una deserción académica está relacionado más al factor económico que a la desintegración familiar, pero que, a pesar de eso, la estabilidad psicológica de los estudiantes se ve afectada por las expectativas y demandas de los padres o representantes y por la mala influencia de terceros. Siendo así se comprobó que la deserción surge con mayor frecuencia en participantes femeninos (71.43\%) que en participantes masculinos (28.57).

\section{DISCUSIÓN}

Cada teoría y aporte de los autores estudiados han permitido relacionar la problemática desde otras ramas de las ciencias sociales, si bien es cierto las circunstancias en las que se presenta la deserción son diferentes para cada individuo por lo que es difícil prevenir; de acuerdo con esto la hipótesis principal ha sido comprobada en su totalidad, además surgen nuevas interrogantes dentro del ámbito social ¿Por qué no se lo detecta en sus inicios?, ¿ Si no hay la presencia de estos factores, se considera como problemática?, ¿El actuar ante este problema es eficiente?; cada una de estas interrogantes es válida; para lo cual se puede responder que la detección de los factores permiten la detección temprana permitiendo abordar el problema principal que dará como consecuencia evitar la deserción temporal o definitiva.

\section{CONCLUSIÓN}

Las diferentes teorías estudiadas han permitido definir la deserción más que un problema institucional, debe ser considerado como un problema social; la deserción es más una consecuencia de otras problemáticas pero que a pesar de ellos no deja de ser un problema. El principal factor que afecta en la continuidad académica se dan en el entorno familiar del cual se derivan otros factores, este mantiene una estrecha relación con los factores socioeconómicos. Como consecuencia surge la deserción académica y otros nuevos problemas como el bajo nivel de persistencia y el limitado desarrollo económico social.

Desde el Trabajo Social en el ámbito de la educación, se aborda la problemática desde la raíz, el cual surge dentro del entorno familiar por factores socioeconómico y otros motivos; en el entorno pedagógico esta la relación entre pares y docentes, el rendimiento académico; pero no se las detecta hasta que el estudiante decide abandonar sus estudios. La intervención de estas problemáticas pretende prevenir la deserción académica a través del apoyo institucional, pero no depende solamente de la institución, sino también, del 
compromiso del representante o padre familia ya que son el principal vinculo que tiene el estudiante.

Ilustración 2. Intervención Trabajo Social ante la Deserción Académica

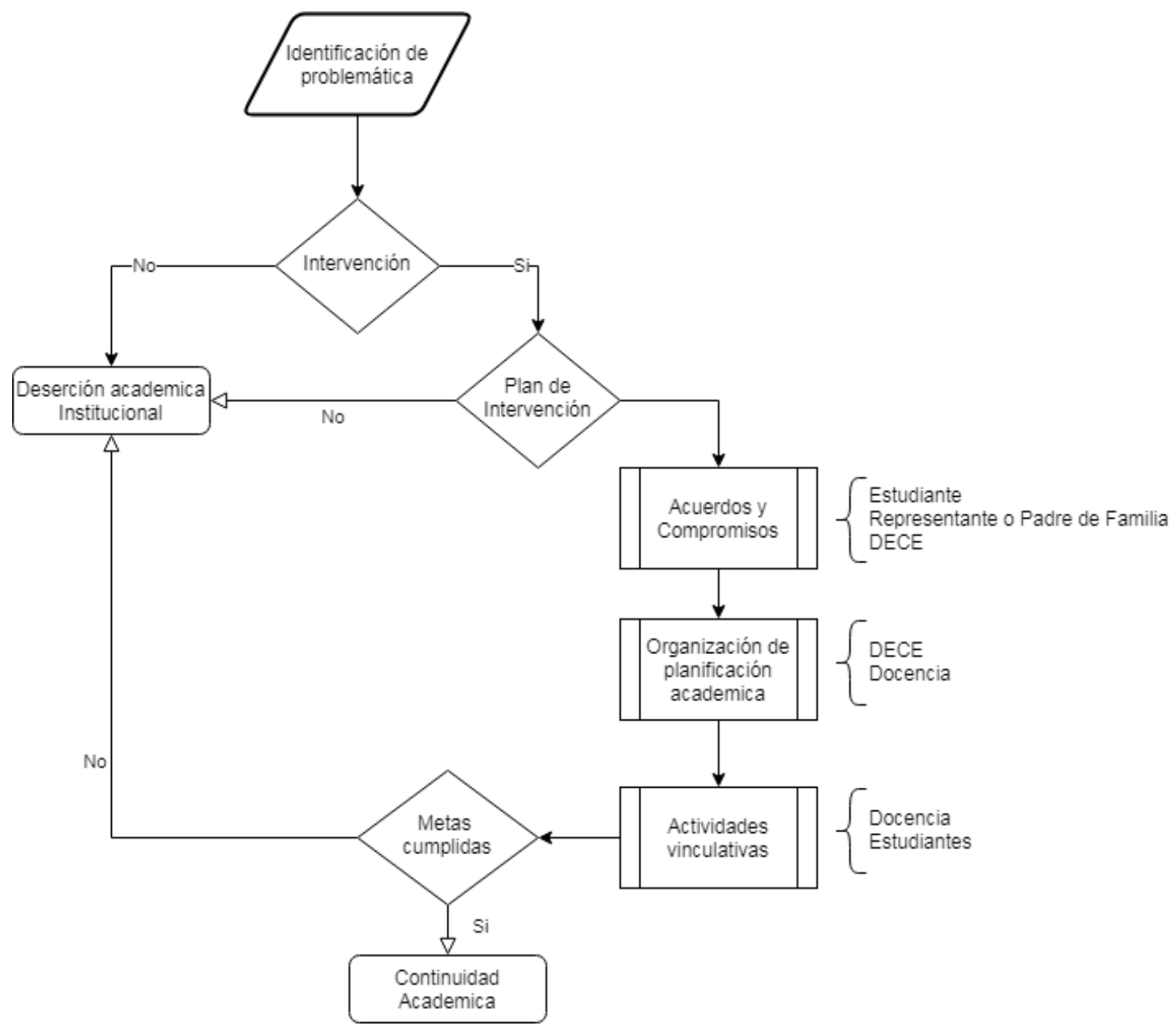

Elaborado por: Arelys Escandón Barnuevo

De acuerdo con la investigación bibliográfica se ha permitido crear una teoría que hemos denominado teoría de influencia de ejes, ya que tanto aspectos económicos como socioculturales los cuales denominaremos como "Eje 1: Factores externos", afectan al "Eje 2: Factores internos" que serían el núcleo familiar y el entorno educativo, dependiendo como influya el eje 1 en el eje 2 podría cambiar los resultados, es decir, si influye positivamente el estudiante logrará la continuidad y excelencia académica, pero si influye de manera negativa se obtendrá la desvinculación o deserción académica 
Ilustración 3. Teoría de influencia de ejes

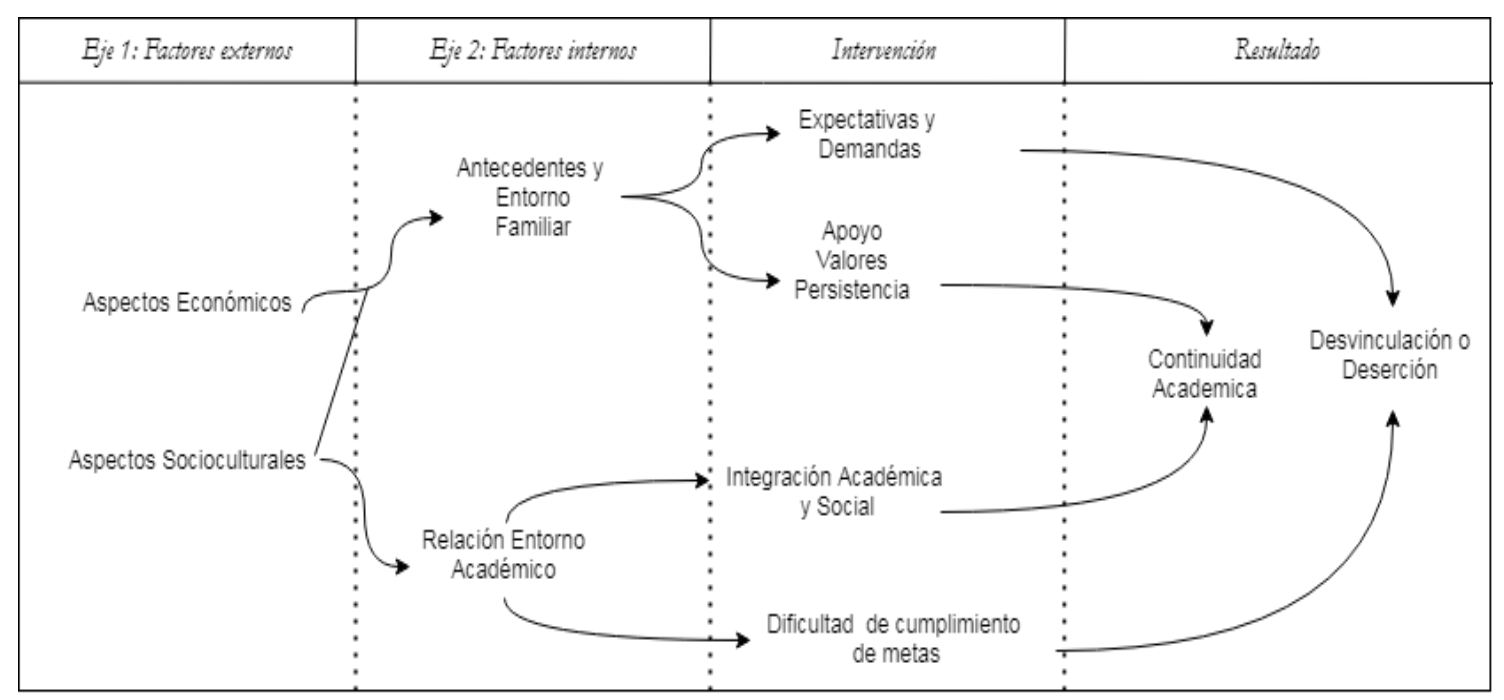

Fuente: (Cardoso \& Verner, 2011), (Brea de Cabral, 2005), (Himmel, 2018.), (SevillaNúñez, Puerta-Chavarría, \& Dávila-Molina, 2010)

Elaborado por: Arelys Escandón Barnuevo

Se considera que la deserción surge más en mujeres que en varones, pero a su vez se pudo comprobar la importancia del problema entre la población; de acuerdo con los encuestados las instituciones educativas deberían dar mayor apertura al diálogo para conocer la opinión de los estudiantes, mientras que los docentes concuerdan que para abordar la deserción de manera efectiva debe mejorar el entorno familiar en el cual se desarrolla el estudiante; por otra se evidenció el poco interés de la población ante esta problemática.

\section{REFERENCIAS BIBLIOGRAFICAS}

Brea de Cabral, M. (2005). Deserción en la educación superior pública en República Dominicana. Revista PsicologiaCientifica.com.

Cardoso, A. R., \& Verner, D. (abr./jun. de 2011). Factores de la deserción escolar en Brasil. El papel de la paternidad temprana, la mano de obra infantil y la pobreza. El trimestre económico, 78(310).

Carnoy, M., Álvarez Méndez, J. M., Moncada, A., Kwong, J., Viñao Frago, A., Fullat, O., . . Forquín, J.-C. (1985). El enfoque sociológico del éxito y el fracaso escolares: desigualdades de éxito escolar y origen social. En Educación y Sociedad (págs. 170-209). AKAL editor. 
Castro Clemente, C., \& Pérez Viejo, J. (2017). EL TRABAJO SOCIAL EN EL ENTORNO EDUCATIVO ESPAÑOL. Revista Castellano-Manchega de Ciencias Sociales, 215-226.

Deserción escolar. (s. f.). Recuperado 29 de diciembre de 2020, de https://www.uaeh.edu.mx/scige/boletin/prepa3/n8/p1.html

Díaz Peralta, C. (2008). MODELO CONCEPTUAL PARA LA DESERCIÓN ESTUDIANTIL UNIVERSITARIA CHILENA. Estudios pedagógicos (Valdivia), 34(2), 65-86.

Espinoza, O., Castillo, D., Gonzales, L. E., \& Loyola, J. (2012). Factores familiares asociados a la deserción escolar en Chile. Revista de Ciencias Sociales, 136-150.

Espinoza-Díaz, O., González, L. E., Cruz-Grau, E. S., Castillo-Guajardo, D., \& LoyolaCampos, J. (2014). Deserción escolar en Chile: un estudio de caso en relación con factores intraescolares. Educación y Educadores, 32-50.

Flores, D. (2010). La escuela como agente de Socialización en los niños. Cuenca, Ecuador.

Girón, L. E., \& González, D. E. (2005). Determinantes del rendimiento académico y la deserción estudiantil. Revista Económica.

Goicovic Donoso, I. (2002). Educación, deserción escolar e integración laboral juvenil. Última Década, 11-52.

Himmel, F. (2018.). Modelo de análisis de la deserción estudiantil en la educación superior. Calidad en la Educación.

Infante, R., \& Parra, L. (2016). Deserción escolar y desarrollo social: Una mirada sobre el Programa "Volver a la Escuela"en Bogota. Revista Educacion y Desarrollo Social, 75-86.

Mendoza Cárdenas, E., \& Zúñiga Coronado, M. (2017). Factores intra y extra escolares asociados al rezago educativo en comunidades vulnerables. Alteridad. Revista de Educación, 79-91.

Ministerio de Educación. (2016). Modelo de Atención Integral de los Departamento de Consejería Estudiantil. Quito: Ministerio de Educación.

Núñez, D. de S. S., Chavarría, V. A. P., \& Molina, J. D. (2010). INFLUENCIA DE LOS FACTORES SOCIOECONÓMICOS EN LA DESERCIÓN ESTUDIANTIL DE 
LA CARRERA DE CIENCIAS SOCIALES. Ciencia e Interculturalidad, 6(1), 7284.

Pacho, F., \& Chiqui, D. (2011). Estudio de las causas de la deserción escolar. Cuenca, Azuay, Ecuador.

Peña, J. C., Soto Figueroa, V. E., \& Calderón Aliante, U. A. (2016). La influencia de la Familia en la Desercion Escolar. Revista Mexicana de Investigación Educativa, 881-889.

Ramírez Martínez, C. (2011). La influencia de la Escolaridad de los padres en el aprendizaje de los niños de primaria. La influencia de la Escolaridad de los padres en el aprendizaje de los niños de primaria. Ciudad del Carmen, Ciudad de México, México.

Rumberger, R. W. (1995). "Dropping out of Middle School: A Multilevel Analy-sis of Students and Schools”. Ameri-can Educational Research Journal., 583-625.

Rumberger, R. W. (01 de mayo de 2001). Why Students Drop Out of School and What Can Be Done. University of California, Santa Barbara.

Ruiz-Ramírez, R., García-Cué, J. L., \& Pérez-Olvera, M. A. (Julio - diciembre de 2014). CAUSAS Y CONSECUENCIAS DE LA DESERCIÓN ESCOLAR EN EL BACHILLERATO. RA XIMHAI, 10(5), 51-74.

Sánchez, D. (2015). La tendencia del abandono escolar en Ecuador: periodo 1994 - 2014. Valor Agregado, 39-57.

Sarmiento Sánchez, C. (2018). Contribución del Trabajo Social en el campo de la Educación. Centro Latinoamericano de Trabajo Social: Nueva Acción Crítica.

Sevilla-Núñez, D. D., Puerta-Chavarría, V. A., \& Dávila-Molina, J. (2010). Influencia de los factores socioeconómicos en la deserción estudiantil de la carrera de ciencias sociales. Ciencia e Interculturalidad, 6(1), 72-84.

Torres, M. (28 de diciembre de 2020). Expreso. La pandemia deja a más de 24.000 niños sin estudiar.

Vásquez, J., Castaño, E., Gallón, S., \& Gómez, K. (2003). Determinantes de la deserción estudiantil en la Universidad de Antioquia.

Venegas, G., Chiluisa, M., Castro, S., \& Casillas, I. (s.f.). La Deserción en la Educación. Boletín Redipe, 6-4. 